\title{
Evaluation of New Voltage Operating Strategies for Integration of Distributed Generation into Distribution Networks
}

\author{
Milana Plecas, Member, IEEE, Simon Gill, Member, \\ IEEE, Ivana Kockar, Member, IEEE \\ Department of Electronic and Electrical Engineering \\ University of Strathclyde, Glasgow, UK
}

\begin{abstract}
With the increasing number of distributed generation connections to distribution networks, the need for better understanding of the distribution network constraints becomes crucial. As distribution networks have not been traditionally designed for two-way power flow, the reverse power flows due to the integration of distributed generation changes voltage profiles and can create significant network management issues related to both thermal and voltage limits. Whilst a large body of theory exists on the management of voltage profiles and the integration of distributed generation into voltage-constrained feeders, there has been limited real world application of these methods to date, in part because network operators are reluctant to undertake significant changes that may affect the reliability of their network. This paper provides a case study of three adjustments to existing management of an $11 \mathrm{kV}$ feeder that are simple and feasible to implement and evaluates their impact on connection capacity for distributed generation including the importance of location. These adjustments are: increased operational upper-voltage levels, simple demand-management, and non-firm connections to manage local voltage constraints.
\end{abstract}

Index Terms-Distributed Generation, Distribution Network, Active Network Management, Voltage Management.

\section{INTRODUCTION}

Traditionally, distribution networks have been passive, designed around centralized power generation with the unique direction of electricity flow from the transmission system to the consumers. However, with the mass integration of renewable generation connected at lower voltage levels, the distribution networks have become active systems with bi-directional power flows. Additional complexity has started to appear caused by voltage rise and constraints on the power flows, which introduce significant network management issues. Therefore, in order to facilitate more distributed generation (DG), Distribution Network Operators (DNOs) need to more actively operate their networks. Active Network Management (ANM) has been developed over the past decade in the UK as one potential solution [1], and has been used to manage thermal limits, allowing significantly cheaper and more timely connections for DGs.

This work is part of the Accelerating Renewable Connections supported by SP Energy Networks and Low Carbon Network Fund, UK.

\author{
Ross Anderson \\ Future Network Department \\ SP Energy Networks \\ Blantyre, UK
}

ANM extends traditional limits on DG capacity of the existing network through real time management of generation (and potentially loads) on the network to match the available network capacity according to Principles of Access (PoA) [1]. There are two types of commercial arrangements:

- 'firm connection' which allows generator to export power to the network at all times, and

- 'non-firm connection' which does not guarantee access to the network at all times but rather stipulates that the generator would need to curtail its output whenever instructed to do so by a DNO to avoid network limits being exceeded.

The first ANM scheme in the UK was installed on Orkney distribution network [2]. Although the Orkney Islands have significant potential for wind generation, the ability of Orkney distribution network to accept further DG connection has been limited by the thermal export capacity of two $33 \mathrm{kV}$ submarine cables connecting the network with the GB mainland. With the islands' demand varying from $8 \mathrm{MW}$ to $32 \mathrm{MW}$; the maximum network DG capacity was $28 \mathrm{MW}$ when applying conventional approaches to network planning which includes a case of minimum demand and a loss of one of the cables. The first round of 'non-firm' connections added 20MW by applying a fast-acting protection system, which trips off the additional generation in the event of a fault on one of the undersea cables. Beyond these $48 \mathrm{MW}$, further DG connections would either require an additional undersea cable to link the network with the UK mainland or active management. Since the reinforcement option would have cost an estimated $£ 30$ million, an ANM scheme with total cost of $£ 0.5$ million was installed instead [2]. By 2013, ANM had enabled the additional capacity of distributed wind generation of around $24 \mathrm{MW}$, by instructing generators to trim their output to maintain thermal limits across the distribution network.

ANM has also been rolled out on the Shetland Islands as a method of managing the stability of the islanded distribution network [3]. On the UK mainland, ANM has been proposed in the areas where the network capacities are close to their limits in terms of DG connections. These are part of a number of Low Carbon Network Fund projects [4], such as Low Carbon Hub [5] and Flexible Plug and Play [6]. However, all of these have focused on the management of network constraints at $33 \mathrm{kV}$ level. The 
management of thermal constraints and especially voltage constraints, which is a potentially more complex issue, has not yet been adequately resolved by UK ANM schemes at $11 \mathrm{kV}$ level. One of the first UK project looking to deploy the ANM scheme at $11 \mathrm{kV}$ level, is the Accelerating Renewable Connections (ARC) project [7]. It investigates different ways of integrating new DG connections to distribution networks that were considered full under existing management strategies. The ARC project explores management of voltage constraints, identifying the best ways to operate voltage constrained $11 \mathrm{kV}$ feeders with large penetration of DGs. In particular, it investigates whether existing operating principles should be changed to facilitate greater firm and non-firm connection capacity.

The integration of DG onto voltage constrained distribution networks, and the subsequent real-time management or optimisation of voltage has a large theoretical literature, for example [8]-[11]. Despite this, implementation of these techniques has not been followed for several important reasons. Firstly, the considerable monitoring and control infrastructures required for actively managing a network must be installed from scratch and secondly, reliance on new technologies can be seen as a risk by DNOs charged with maintaining safety and security of supply. Finally, many proposed schemes require wholesale changes in operating policy rather than incremental adjustments.

This paper presents work that seeks to evaluate how incremental changes in the existing operating principles and the location of generators can increase the capacity of the $11 \mathrm{kV}$ networks to connect DGs. Three different strategies are investigated: (i) relaxing the upper voltage operational limit at the DG point-of-connection, (ii) increasing demand, for example through demand management, and (iii) the utilisation of non-firm agreements to manage the voltage constraints in a similar way to that used with management of thermal constraints. Each of these can be implemented individually, or in combination with the others, or a wider active management of Smart Grid scheme.

\section{DESCRIPTION OF THE MODEL}

The feeder model used in this paper, shown in Fig. 1, was developed in DIgSILENT PowerFactory, a software tool for power system analysis [12]. The model represents a real $11 \mathrm{kV}$ feeder operated by a UK DNO.

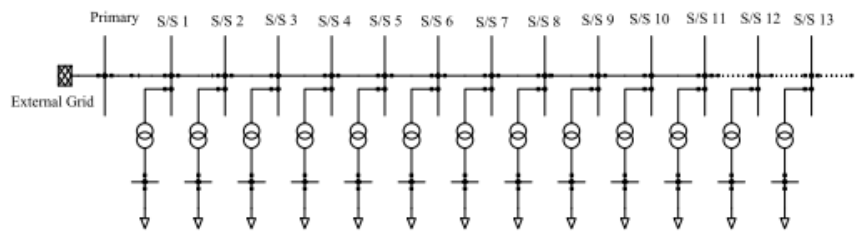

Fig. 1. The model of the $11 \mathrm{kV}$ feeder.

The feeder is connected to a $33 \mathrm{kV} / 11 \mathrm{kV}$ primary substation with the external grid acting as the swing bus. It is $17.9 \mathrm{~km}$ long consisting of $9.5 \mathrm{~km}$ of underground cables (solid lines), and $8.4 \mathrm{~km}$ of overhead cables (dotted lines). It supplies thirteen secondary substations, named S/S 1-13. Each secondary substation (S/S) includes an $11 \mathrm{kV} / 433 \mathrm{~V}$ transformer and a balanced Low Voltage (LV) load. The size of transformers varies from $0.3 \mathrm{MVA}$ to $1 \mathrm{MVA}$ with the impedance of $4.75 \%$.

The analysis carried out in this paper was based on historic voltage and demand data for the period April-June 2014. These data consist of line-to-line voltages measured at the $11 \mathrm{kV}$ bus bar at the primary substation and $\mathrm{LV}$ active and reactive power per each phase at each $\mathrm{S} / \mathrm{S}$.

\section{EXISTING OPERATING PRINCIPLES}

In the UK, DNOs must ensure that the voltage across their network stays within statutory limits, which, for the $11 \mathrm{kV}$ network, are $+/-6 \%$. This means that voltages at all points must stay within the range $10.34-11.66 \mathrm{kV}$ [13]. However, as very limited monitoring equipment is connected at either $11 \mathrm{kV}$ level or lower voltage levels, and no real time control actions are possible, DNOs generally apply more stringent operational limits [14]. When considering the connection of DG to an $11 \mathrm{kV}$ feeder a DNO carries out studies to ensure that: (i) at periods of low demand, DG not overload the thermal limits of the feeder; and (ii) under all expected operating conditions, voltage limits across the feeder are maintained within operational (rather than statutory) limits.

A typical operational voltage regime involves limiting the voltage at the DG point-of-connection to a maximum of $11.25 \mathrm{kV}$ under worst-case conditions [7]. The feeder is usually operated with the primary voltage set slightly higher than the nominal value as historically voltages will reduce along the feeder. A typical value for the $11 \mathrm{kV}$ voltage at the primary substation is $11.2 \mathrm{kV}$. These choices are developed by DNOs through engineering experience and knowledge of the maximum expected voltage drops across the $11 \mathrm{kV}$ and $\mathrm{LV}$ networks.

Connection of DG at $11 \mathrm{kV}$ level is currently limited to only 'firm connection' agreements, meaning that the total firm capacity should not exceed the total generation that can be injected into the $11 \mathrm{kV}$ network under conditions of minimum demand. The determination of whether to award a 'firm connection' to a generator at a particular location on an $11 \mathrm{kV}$ feeder depends on the studies of the voltage at the point-ofconnection of the generator. Such studies typically use a procedure similar to the following:

- Set the voltage at the primary substation to $11.2 \mathrm{kV}$ based on typical operation of the primary On Load Tap Changer.

- Set all demand to the minimum expected level.

- Set existing firm generation to its rated output.

- Set the proposed generation output to its rated capacity.

- Run a power flow simulation to calculate the point-ofconnection voltage for the generator.

- If the point-of-connection voltage exceeds $11.25 \mathrm{kV}$, the generator is not allowed to connect.

This procedure has some limitations. For example, it does not take full account of the effect of increased generation on the 
primary voltage within the On Load Tap Changer dead band. However, when compared with operation experience the results produced are realistic, and importantly, form the basis of realworld decisions. A further observation is that real-world decision making such as this does not attempt to prioritise generation at one location over another in order to approximate an electrically optimal solutions; the geographical location of schemes that apply for network connection is not within the network company.

To illustrate the capacity for firm DG connections that is available at different locations on the case study feeder, and how the connection of one generator affects the remaining capacity for further connection others, a methodology is developed to find the maximum capacity of distributed generation that maintains the thermal and voltage limits under the historic conditions. Two different scenarios are investigated: (i) when only one DG is connected to the feeder and (ii) when multiple DGs are connected to the feeder. The process is as follows:

- Primary substation voltage is set to the value recorded historically.

- Real and reactive demands are set to the value recorded historically at each S/S.

- DG, operating at unity power factor, is added to a single $\mathrm{S} / \mathrm{S}$ and its output is increased until either a thermal limit is reached or the voltage at the point-of-connections reaches $11.25 \mathrm{kV}$.

\section{A. Available generation capacity when only one DG is connected to the feeder}

The calculation is carried out for three demand levels: low (LD), medium (MD) and high demand (HD). It is first conducted with one DG connected and repeated for all secondary substations to identify the firm capacity available at each S/S independent of the others. Note that primary voltage is set to the value recorded historically at the time of each demand scenario rather than being set nominally to $11.2 \mathrm{kV}$ as often used by DNOs.

The available DG capacity for the three demand conditions along the length of the feeder is shown in Fig. 2. In all cases, the capacities at the secondary substations closest to the primary, S/S 1-2, are constrained by thermal limits. The limiting power flow along the feeder is approximately 4.3MVA, and the maximum thermally constrained DG capacity is approximately the sum of 4.3MW and current demand on the feeder.

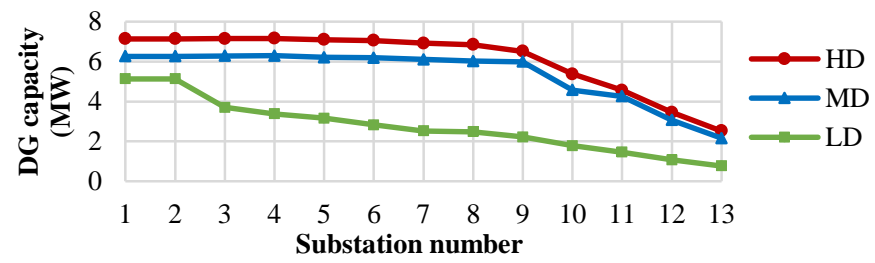

Fig. 2. The capacity profile along the feeder for three demand scenarios when only one DG is connected to the feeder.

In the LD case, which represents the worst-case conditions and therefore sets the firm limit, all other substations are voltage constrained. The gap between the LD and HD case therefore represents the additional capacity that may be available for nonfirm generators to access at time where demand is higher than minimum.

\section{B. Available generation capacity when multiple DGs are connected to the feeder}

The available generation capacity calculated at each $\mathrm{S} / \mathrm{S}$ in the previous section is independent of the capacity calculated at all other substations, as only one DG is connected to the feeder at all time. The connection of one DG immediately reduces the remaining capacity for subsequent DGs. Where the binding limit is thermal, the reduction in remaining capacity is approximately equal to the capacity of the first generators, however, where voltage limits are binding this is not true.

To investigate the effect of interaction between two DGs, the previous methodology is adjusted to calculate the remaining DG capacity after the connection of a $0.5 \mathrm{MW}$ firm generator (FDG) representing a typical high capacity-factor controllable generators (assumed here to be generating at full capacity in all cases). The connection of the FDG is modelled at three locations:

- S/S 1 (FDG1): close to the primary,

- $\mathrm{S} / \mathrm{S} 7$ (FDG2): in the middle of the feeder, and

- S/S 13 (FDG3): at the end of the feeder.

The effect on the remaining DG capacity across the feeder for low demand (worst-case conditions) is illustrated in Fig. 3. It compares the initial capacity for DG (as calculated in the previous section) with the remaining capacity when the first firm generator is connected at each of the three potential locations.

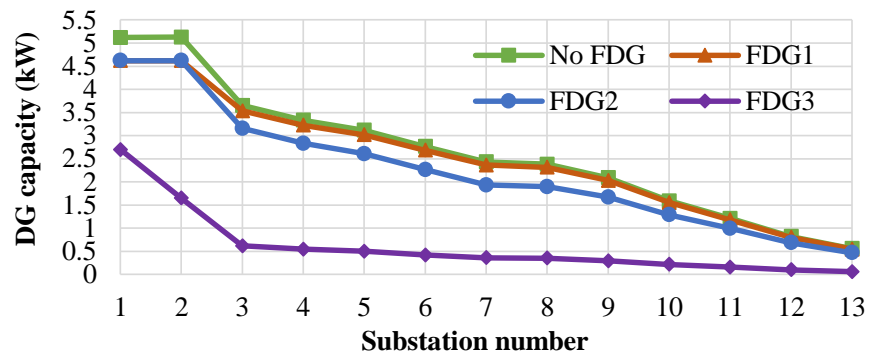

Fig. 3. The capacity profile along the feeder for low demand scenario when multiple DGs are connected to the feeder.

The addition of the firm generator at either S/S 1 (FDG1) or S/S 7 (FDG2) reduces the DG capacity at thermally constrained substations, S/S $1-2$ by approximately $0.5 \mathrm{MW}$ as expected. The firm generator effectively reduces the feeder demand by $0.5 \mathrm{MW}$ and therefore reduces the available thermal capacity by $0.5 \mathrm{MW}$.

However, the connection of the firm generator at S/S 13 reduces the DG capacity at S/S 1-2 by significantly more. This is because the voltage at $\mathrm{S} / \mathrm{S} 13$ is now close to $11.25 \mathrm{~V}$ and addition capacity anywhere else on the feeder further pushes the S/S 13 voltage up to its limit. It is the $\mathrm{S} / \mathrm{S} 13$ voltage limit that is binding, rather than the thermal export limit at the start of the feeder.

In the case of voltage-constrained substations, it is not only the size of the firm generator but its location that affects the 
remaining capacity. It can be seen that the addition of the firm generator at $\mathrm{S} / \mathrm{S} 1$ has an almost negligible effect on capacity at voltage-constrained substations, S/S 3-13. This means the feeder can support the original DG capacity at these locations and the $0.5 \mathrm{MW}$ firm generator. The reason is that the firm generator is close to the primary where the voltage is fixed and the injection of $0.5 \mathrm{MW}$ at $\mathrm{S} / \mathrm{S} 1$ therefore does not significantly affect the voltage profile across the feeder.

Whilst these results agree with existing work that study optimal connection arrangement, it is important to remember that such optimisation is often not feasible with DNOs asked to accept and reject developer proposals. However, in evaluating a particular request for connection, a DNO must keep an eye to potential future developments, and the impact of allowing current projects to connect on the remaining capacity for others.

\section{STRATEGIES FOR OPERATING VOLTAGE CONSTRAINED $11 \mathrm{KV}$ FEEDER}

This section presents results of investigations into three possible strategies for managing voltage profiles along the $11 \mathrm{kV}$ feeder in order to allow more DG connection capacities.

\section{A. The effect of raising the point-of-connection voltage limit}

The analysis carried out in the previous section was based on a DG point-of-connection limit of $11.25 \mathrm{kV}$, which is an operational limit taken to ensure that voltage further down the network, including both the $11 \mathrm{kV}$ and LV sections, will remain within statutory tolerances under all conditions. To investigate the effect of raising this limit, the power flow study simulations were repeated using the same historic demand profiles with the pointof-connection limit raised up to $11.4 \mathrm{kV}$ with steps of $0.5 \mathrm{kV}$.

The effects of raising the point-of-connection voltage limit during the low demand are illustrated in Fig. 4 and Fig. 5 shows the increase in DG capacity for each voltage limit.

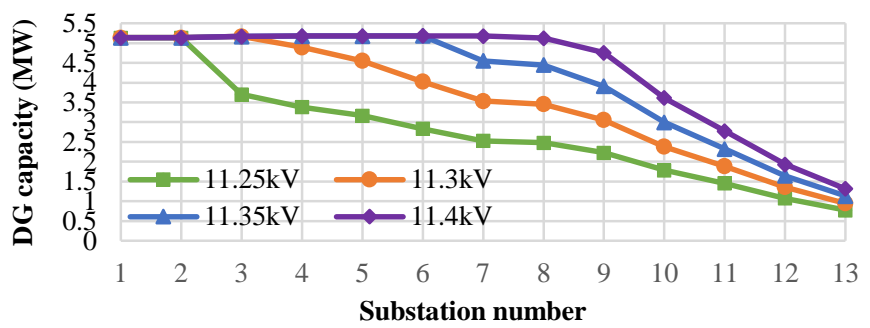

Fig. 4. The capacity profile along the feeder for low demand scenario with different point-of-connection voltage limits.

While thermally constrained capacities, at S/S 1-2, remain unchanged, voltage constrained capacities, S/S 3-13, increase linearly before reaching their thermal constraint. At the end of the feeder, the increase is relatively smaller for all point-ofconnection limits due to the voltage constraint and therefore there are no cases of thermal constraint violations.

This shows that the increase in the operational voltage limit at the point-of-connection allows greater capacity to connect to any substation that is voltage constrained, whilst substations that were originally thermally constrained generally remain so under the new voltage conditions.

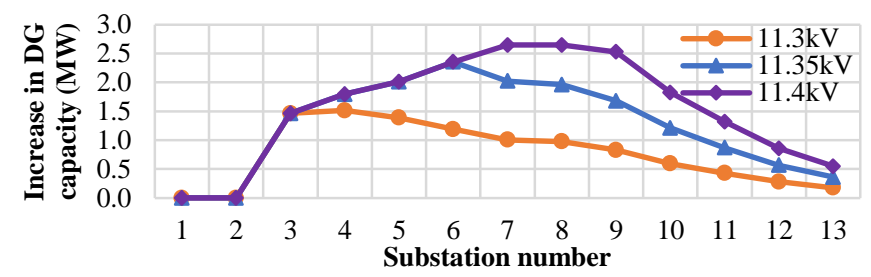

Fig.5. Increase in DG capacity along the feeder for low demand scenario for raised point-of-connection voltage limits.

This represents a simple step for increasing DG capacities, and continues to maintain a buffer between the operational limit and the statutory $11.66 \mathrm{kV}$ limit. However, ensuring that such a change in policy is safe and secure will require detailed studies to ensure that under all credible operating conditions voltages at all un-monitored points of the $11 \mathrm{kV}$ and associated $\mathrm{LV}$ feeders are within limits.

\section{B. The effect of increasing demand}

Instantaneous demand increases can be created either through the development of new electrical demand, for example converting a non-electrical energy demand, such as oil-heating to electric heating, or alternatively through demand-side involvement, i.e. the use of flexible demand.

If demand and generation are on the same site, an increase in real power demand during a particular time-step will create a 1:1 increase in capacity for DG. However, additional demand may be located elsewhere on the feeder or it can be spread across multiple secondary substations. Therefore, a methodology has been developed to identify the increase in DG capacity created at one $\mathrm{S} / \mathrm{S}$ if demand is increased at a different $\mathrm{S} / \mathrm{S}$. The methodology increases demand by $100 \mathrm{~kW}$ at different $\mathrm{S} / \mathrm{S}$ and calculates the increase in DG capacity at a particular $\mathrm{S} / \mathrm{S}$.

The results for DG capacities at four secondary substations during a single time-step are shown in Fig. 6. Each line represents the increase of DG capacity at a particular $\mathrm{S} / \mathrm{S}$ when demand is added at at each of the 13 substations. The ' $\mathrm{S} / \mathrm{S} 2$ ' line shows that an additional $100 \mathrm{~kW}$ of demand at any substation allows an additional $100 \mathrm{~kW}$ of DG capacity. The capacity of DG at S/S 2 is thermally constrained and therefore additional demand anywhere on the feeder reduces reverse power flow from the feeder to the primary substation on approximately 1:1 basis, with small variations due to changes in losses and reactive power flows. Other locations shown in Fig. 6 are voltage constrained and therefore the effectiveness of additional demand at creating further DG headroom depends on its location. For example, for DG located at S/S 13, additional demand located closer to the primary has a reduced effect; that is $100 \mathrm{~kW}$ of additional demand would lead to less than $100 \mathrm{~kW}$ of extra DG capacity. 


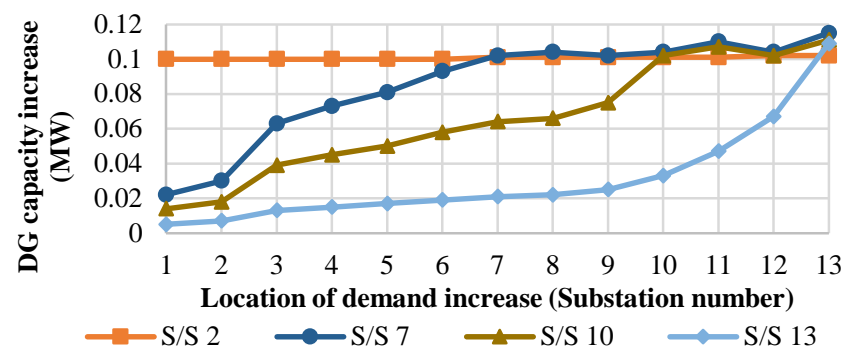

Fig. 6. DG capacity increase due to a demand increase during a single time-step.

The results shown in Fig. 6, replicated at all time-steps can be summarised as follows:

- Additional demand at the same S/S as a DG raises capacity for that DG on a 1:1 basis.

- For substations where DG is thermally constrained, the additional demand leads to approximately a $1: 1$ increase in DG capacity regardless of the location of the demand.

- Where DG capacity is voltage constrained, additional demand located closer to the primary than the particular DG, has smaller effect on DG capacity than 1:1. Every unit of additional demand creates less than one unit of extra DG capacity.

- Where DG capacity is voltage constrained and the additional demand is located further from the primary than the DG unit, the additional demand creates approximately a 1:1 increase in DG capacity.

\section{Non-firm connection agreement}

To date, a number of ANM schemes have used non-firm connection agreements to manage thermal limits. However, the same principle can be applied to voltage constrained feeders where a DG with 'non-firm connection' would need to curtail its output whenever instructed to do so by a DNO to avoid both thermal and voltage constraint violations.

For a given size of non-firm generator wishing to connect at a particular S/S, the expectation of curtailment can be estimated using historical times-series of available generation and the available network capacity. The available generation time-series depends on the generation technology and can be modelled by scaling historic time-series from an existing local generator to the installed capacity of the proposed non-firm generator.

To illustrate this method, a normalised available wind profile, $P_{\text {norm wind }}(t)$ and the historic network demand and voltage data for April-June 2014 are used. The study identifies the capacity of DG that can connect at each location whilst experiences different amount of curtailment of its potential output. This is referred to as $P_{c \%}^{n f}$, meaning the DG capacity available for non-firm generator with $c$ of its output curtailed. The comparison of the available wind generation against the total available network capacity, $P_{\text {net }}(t)$, taking account of thermal and voltage limits, for a 10 day period is shown in Fig. 7. Where the wind generation is greater than the network capacity, generation curtailment is required to reduce output to match network capacity.

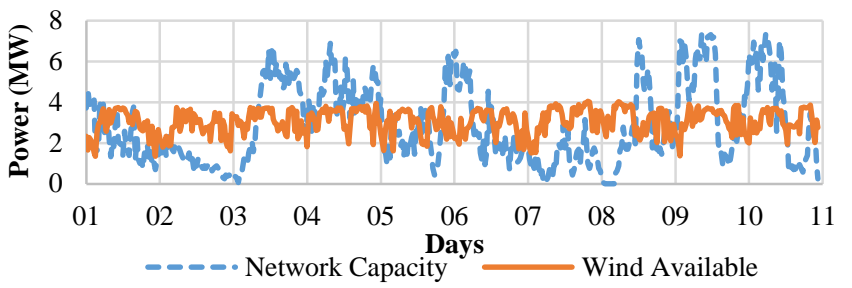

Fig. 7. Comparison of the network capacity against available wind generation.

In order to find the non-firm capacity at each $\mathrm{S} / \mathrm{S}$ for a particular level of curtailment, assuming generation up to the firm limit is already installed, the firm capacity, $P_{\text {firm cap }}$, available at each $\mathrm{S} / \mathrm{S}$ should be calculated. The firm capacity, strictly defined, is the lowest capacity calculated during any time-step. However, using real historical data includes period of fault conditions and other abnormal operating conditions. Therefore, in order to remove these, the firm capacity limit in this study is based on the $99^{\text {th }}$ percentile meaning the network capacity available for $99 \%$ of the time whilst maintaining operational limits.

To calculate the non-firm capacity at each S/S that will experience curtailment level, $c$, the following method is used:

1. Calculate the firm generation profile by scaling the normalised available wind profile:

$$
P_{\text {firm }}(t)=P_{\text {firm cap }} \times P_{\text {norm wind }}(t)
$$

2. Calculate the remaining network capacity at each time step available to non-firm generation:

$$
P_{n f}^{\max }(t)=P_{n e t}(t)-P_{\text {firm }}(t)
$$

3. Start with a small non-firm capacity of $P_{c \%}^{n f}=0.1 \mathrm{MW}$. Calculate the available non-firm generation profile by scaling the normalised wind profile:

$$
P_{n f}^{\text {available }}(t)=P_{c \%}^{n f} \times P_{\text {norm wind }}(t)
$$

4. Calculate how much curtailment is required for each timestep to stay within the network limits.

$$
P_{n f}^{\text {curt }}(t)=\left\{\begin{array}{c}
0 \text { if } P_{n f}^{\max }(t)>P_{n f}^{\text {available }}(t) \\
P_{n f}^{\text {available }}(t)-P_{n f}^{\max }(t) \text { otherwise }
\end{array}\right.
$$

5. Calculate the fraction of curtailed non-firm generation curtailment across the study.

$$
P_{n f}^{\text {curt }}=\sum_{t} \frac{P_{n f}^{\text {curt }}(t)}{P_{n f}^{\text {available }}(t)}
$$

6. If the fraction of curtailed non-firm generation is less than $c$, increase $P_{c \%}^{n f}$ by $0.1 \mathrm{MW}$ and repeat steps 3-6.

The process 1-6 is repeated for different curtailment levels, $c$, in the range $10-50 \%$ and the capacities connected at each $\mathrm{S} / \mathrm{S}$ based on the three months analysis are shown in Fig. 8.

The results show that the greatest opportunity for non-firm capacity is in the middle regions of the feeder. Large firm capacities, combined with relative proximity to the voltagecontrolled bus, limit the opportunity for non-firm connections close to the primary substation. Towards the end of the feeder, 
available capacity is relatively small due to the large electrical distance from the nearest point of voltage control.

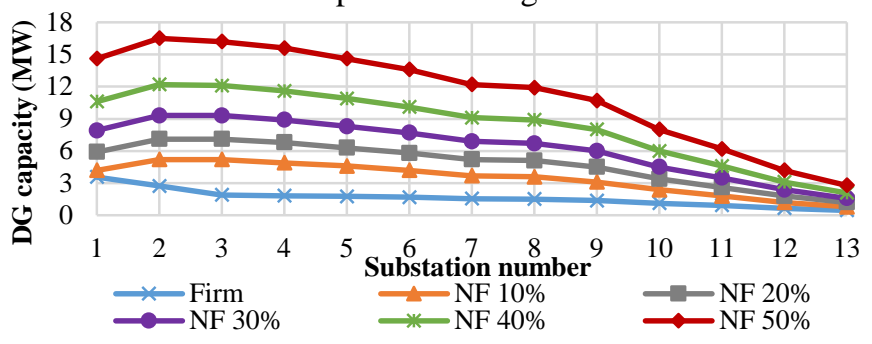

Fig. 8. Firm and non-firm capacity estimates.

\section{DISCUSSION AND CONCLUSIONS}

The work presented in this paper presents the evaluation of different strategies for operating a voltage constrained $11 \mathrm{kV}$ feeder to allow additional DG connections beyond that which can connect under existing operational conditions. A key finding of the paper is that relatively simple interventions, such as the raising of existing operational levels, can increase the level of DG that can be allowed to connect. These strategies would allow an incremental process to be adopted to move from a wholly 'Fitand-Forget' approach to full active management of $11 \mathrm{kV}$ voltage profiles:

- Raising the point-of-connection voltage limit has the potential to increase DG capacity substantially. For example, at $\mathrm{S} / \mathrm{S} 5$ in the example studied, a $150 \mathrm{~V}$ increase in the operational limit from $11.25 \mathrm{kV}$ to $11.4 \mathrm{KV}$ can approximately double the firm capacity.

- Additional demand can create additional capacity for a DG. This demand, in order to be effective at managing voltage constraints, needs to be connected either at the same location as the DG, or further away from the primary. Therefore, encouraging the connection of new demand towards the end of the feeder is a simple way of increasing DG connections, where this can be shown not to cause low voltage problems during periods of low DG output.

- The use of the non-firm connection agreements and active management of generator output against a fixed DG pointof-connection can increase the capacity and total energy yield from DG in a similar way to schemes designed to manage thermal limits. In the example case, a $10 \%$ curtailment level is commensurable with a doubling of capacity in the middle regions of the feeder. Greater curtailment levels are likely to require more careful economic analysis on the part of the developer, but can further increase capacity and annual energy yield from connected DG.

This paper has presented a single feeder case study. However, it will be important to extend these studies to wider $11 \mathrm{kV}$ networks in general. In particular, it will be important to extend this to consider the impact of On Load Tap Changer operation and the interaction between multiple feeders fed from a particular primary substation.

\section{ACKNOWLEDGMENT}

The authors gratefully acknowledge the contributions of SP Energy Networks.

\section{REFERENCES}

[1] Energy Networks Association. (2015, July). Active Network Management Good Practice Guide. [Online]. Available: http://www.energynetworks.org/assets/files/news/publications/1500 205 ENA ANM report AW online.pdf

[2] Smarter Grid Solutions (2013). Island Renewables Integration. [Online]. Available: http://www.smartergridsolutions.com/media/ 51942/orkney_case-study_uk_final.pdf.

[3] S. Gill, M. Dolan, A. Emhemed, I. Kockar, M. Barnacle, G. Ault, and C. Mathieson, "Increasing renewable penetration on islanded networks through active network management: a case study from Shetland," Renewable Power Generation, IET, vol. 9, no. 5, pp. 453465, July 2015.

[4] Ofgem. (2012, Sep.). Low Carbon Networks Fund. [Online]. Available: https://www.ofgem.gov.uk/electricity/distributionnetworks/network-innovation/low-carbon-networks-fund.

[5] Western Power Distribution. (2015, May). Low Carbon Hub project close down report. [Online]. Available: http://www.smarternetworks .org/Files/Low_Carbon_Hub_151022125750.pdf.

[6] UK Power Networks. (2011, Nov.). Flexible Plug and Play close down report. [Online]. Available: http://innovation.ukpower networks.co.uk/innovation/en/Projects/tier-2-projects/Flexible-Plug -and-Play-(FPP)/Project-Documents/Close-Down-Report_Final.pdf.

[7] Scottish Power Energy Networks. (2012, Nov.). Accelerating Renewable Connections Full Submission. [Online]. Available: http://www.smarternetworks.org/Files/ARC_130115123252.pdf.

[8] N. S. Rau and Yih-Heui Wan, "Optimum location of resources in distributed planning," IEEE Transactions on Power Systems, vol. 9, no. 4, pp. 2014-2020, Nov. 1994.

[9] K. Nara, Y. Hayashi, K. Ikeda and T. Ashizawa, "Application of tabu search to optimal placement of distributed generators,"Power Engineering Society Winter Meeting, 2001. IEEE, Columbus, $\mathrm{OH}$, pp. 918-923 vol.2., 2001.

[10]D. Singh, D. Singh and K. S. Verma, "Multiobjective Optimization for DG Planning With Load Models," IEEE Transactions on Power Systems, vol. 24, no. 1, pp. 427-436, Feb. 2009.

[11]L. F. Ochoa and G. P. Harrison, "Minimizing Energy Losses: Optimal Accommodation and Smart Operation of Renewable Distributed Generation," IEEE Transactions on Power Systems, vol. 26, no. 1, pp. 198-205, Feb. 2011.

[12]DIgSILENT PowerFactory 15.2. [Online]. Available: http://www.digsilent.de/index.php/downloads.html.

[13] The Electricity Safety, Quality and Continuity Regulations 2002. (2003, Jan.), p. 14, 27. (3)(c). [Online]. Available: http://www.legislation.gov.uk/uksi/2002/2665/pdfs/uksi_20022665 _en.pdf.

[14] D. Hollingworth, EA Technology. (2014, Dec.). Voltage Control Policy. [Online]. Available: http://www.networkrevolution.co.uk/ wp-content/uploads/2014/12/CLNR-L257-CLNR-Voltage-ControlPolicy-v1.0.pdf. 